\title{
The role of nutraceutical medications in men with non bacterial chronic prostatitis and chronic pelvic pain syndrome: A prospective non blinded study utilizing flower pollen extracts versus bioflavonoids
}

\author{
Angela Maurizi ${ }^{1}$, Francesco De Luca ${ }^{1}$, Antonino Zanghi ${ }^{2}$, Emy Manzi ${ }^{3}$, Costantino Leonardo ${ }^{1}$, \\ Michele Guidotti ${ }^{1}$, F.P. Antonaccio ${ }^{1}$, Valerio Olivieri ${ }^{4}$, Carlo De Dominicis ${ }^{1}$ \\ ${ }^{1}$ Department of Gynaecological and Urological Sciences, Sapienza University of Rome, Rome, Italy; \\ 2 Dipartimento di Medical and Surgical Sciences and Advanced Technologies G.F. Ingrassia, University of Catania, Catania, Italy; \\ ${ }^{3}$ Department of General Surgery, Santa Scolastica Hospital, Cassino (FR), Italy; \\ ${ }^{4}$ Division of Urology, Ivrea Hospital - ASL TO4, Ivrea, Turin, Italy.
}

\begin{abstract}
Summary
Introduction: Chronic prostatitis (CP)/chronic pelvic pain syndrome (CPPS) represents a challenge for the urologist, since the therapeutic efficacy does not always result in a satisfactory quality of life for the patients. Often the side effects of the medications used (antiinflammatories, antibiotics, alpha blockers) far outweighs the benefits gained with their admission. The choice of nutraceutical medications is preferred for their effectiveness, that has been accepted and proven by the scientific community, and for the low incidence of side effects.

The objective of this study to compare the therapeutic efficacy of the flower pollen extracts (Deprox ${ }^{\circledR}$ ) versus Bioflavonoids in terms of reduction of symptoms, and in the average waiting time of the variation of the National Institute of Health Chronic Prostatitis Symptom Index (NIH-CPSI), and to evaluate the quality of life improvement of the patients affected by CP/CPPS. Methods: Among the 68 patients presented with prostatic symptoms to the Hospital "Umberto I" in Rome, Italy between March 2016 and June 2016, 54 patients met the clinical diagnosis of CP/CPPS (class IIIa or IIIb according to the NIH classification). The patients were assigned to either treatment with Deprox ${ }^{\circledR}$ or quercetin based on a randomization scheme previously determined.The NIH- CPSI, IPSS, QoL questionnaires were administered. Every patient underwent bacterial cultures and trans-rectal ultrasound.

Results: There was a statistically significant improvement of the NIH-CPSI score and QoL in the Deprox ${ }^{\circledR}$ group $(p=<0.0001$ and $p=0.003$ respectively). The average waiting time of the variation of the National Institute of Health Chronic Prostatitis Symptom Index (NIH-CPSI) was statistically significant ( $p=$ 0.0019 ). In the absence of efficacy of the "conventional" medications, which also carries significant side effects, the dietary supplements may represent a valid alternative.

Conclusions: DEPROX ${ }^{\circledR}$ has demonstrated a significant improvement of the symptoms and quality of life of patients diagnosed with by CP/CPPS. Furthermore, there was a statistical difference in the average waiting time of the variation of the NIH-CPSI) score without side effects as compared to the bioflavonoids complex with quercetin.
\end{abstract}

KEY WORDS: Chronic prostatitis; Chronic pelvic pain syndrome; Prostatic benign diseases; Inflammation; Pollen extracts.

Submitted 3 July 2018; Accepted 19 August 2018

\section{INTRODUCTION}

In 1995 the National Institute of Health (NIH) proposed a classification of the prostatitis now internationally accepted (1).

The overall incidence of prostatitis in Italy results to be about 13.8\% (2). Based on the literature available data, $5 \%$ of the prostatitis belongs the categories I and II.

The antibiotics treatment, in general, results in a good response with resolution of symptoms. The majority of the prostatitis, however, is represented by the category III type. This is a therapeutic challenge for the practitioners.

Following a first episode of prostatitis, the likelihood of subsequent episodes is very high, ranging from $20 \%$ to $50 \%$ in proportion to the age of the subject. Prior to establish a possible treatment protocol an accurate medical history and physical examination should be carried out. This obviously includes an accurate digital rectal examination. It is also mandatory to administer the NIH-CPSI and the International Prostate Symptom Score (I-PSS) questionnaires and to perform the Meares and Stamey test (3), uroflowmetry, urethral swap test, semen culture, total PSA and a transrectal ultrasonography. In spite of the fact that type III prostatitis is by definition non-bacterial, in many cases the antibiotics administration has resulted in a significant improvement of the symptoms. In fact, the literature supports the use of antibiotics in these cases $(4,5)$. In both cases bacterial and non-bacterial prostatitis alfa blockers can be added to the antibiotics. Modern naturopathic doctors however believe that the prescription of antibiotics for chronic pelvic pain syndrome (CPPS) can do more harm than good when bacteria has not been identified.

However, CPPS is linked to a high rate of treatment failure and patient's frustration due to their unclear etiology and complexity of symptoms. The primary objectives, addressing this condition, are the mitigation of the symptoms, the improvement of the quality of life, minimizing the side effects of the medical treatment. 
Phytotherapeutic agents remains then a suitable choice, especially for their low or absent side effects. However, there are few prospective and controlled studies to support their use $(6,7)$. Several studies demonstrated that a complex of flower pollen extract is able to produce a persistent improvement in symptoms of CPPS with a significant reduction of the NIH-CPSI index $(8,9)$.

A phase II study by Cai et al. demonstrated that the flower pollen extract, in association with vitamins, improved significantly the symptoms, the pain, and the QoL score of patients with non-inflammatory Chronic Prostatitis (CP)/CPPS, without severe side effects (10). Additional studies have demonstrated that several other extract might be able to treat CP/CPPS.

Bioflavonoids are a family of polyphenolic molecules, of which the quercetin is a representative. Quercetin has a theoretical benefits for patients with an ongoing inflammatory or ischemic process of the prostate, mechanisms, which are recognized to be the basis of CPPS. Furthermore, the usually suggested diet poor in bioflavonoids (green tea, caffeine, red wine), quercetin in particular, could worsen the symptoms of CP/CPPS (11). The administration of bioflavonoids is then indicated in order to improve the quality of life of CP/CPPS patients. Also, the bioflavonoids have been shown to play a key role in the inhibition of prostatic cancer cells in vitro (19).

The objective of this study is to assess the efficacy and tolerability of the pollen extract compared to the quercetin in patients with CP/CPPS. The evaluation of the quality of life and the safety of the active principle represent the secondary endpoints of the study.

\section{Materials ANd MEthods}

We performed a non-sponsored phase I-II study in a single urological institution. The study was conducted according to Good Clinical Practice guidelines and the ethical principles of the Declaration of Helsinki. Before the beginning of the study, all participants signed a written informed consent.

Between March 2016 to June 2016, all consecutive patients presenting with clinical diagnosis of CP/CPPS (classes IIIa or IIIb) were recruited in a single urological institution. The study was designed in accordance with the CP/CPPS clinical trial guidelines described by Chronic Prostatitis Collaborative Research Network NIH (12). All the eligible patients completed and returned also the base questionnaires (IPSS, QoL, NIH-CPSI). Following the guidelines of the European Association of Urology (EAU) every patient underwent an thorough urological examination and Meares-Stamey test (13). All the patients

\section{Figure 1.}

who qualified for the study were randomized to either receive flower pollen extract (DEPROX $500^{\circledR} \mathrm{mg} 2$ capsules once a day), or quercetin (500 mg twice a day) for 4 weeks.

The use of bioflavonoids was selected based on the results of Shoskes et al. (15). The patients enrolled were blinded to the type of treatment. A telephone follow up was then conducted 10 days after the treatment to ensure the correct compliance, whilst an outpatients follow up was carried out 30 days after the treatment consisting in a new set of questionnaires plus a urological examination and repeat microbiology cultures.

\section{Inclusion/exclusion criteria}

Inclusion criteria for the enrolment were defined as follows: the presence of persistent pelvic pain for at least 3 months, in the 6 months preceding the study according to the EAU guidelines; NIH-CPSI pain score greater than 7 and a negative Meares-Stamey test (14).

Flowchart of the study.

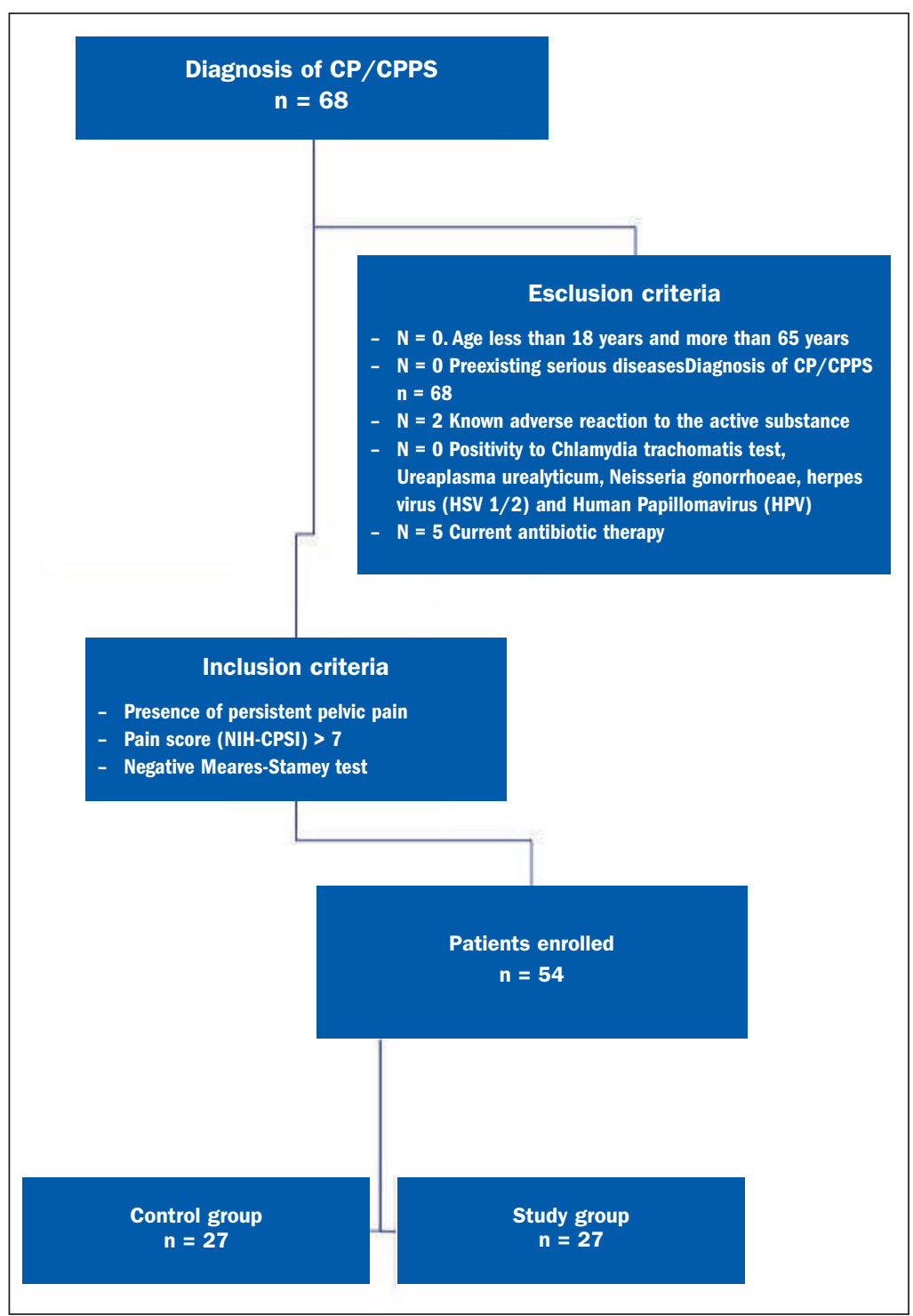


Exclusion criteria were defined as follows: age less than 18 years old and more than 65 years old; anatomical abnormalities of the urinary tract system; additional urologic diseases; post-void residual urine volume (PVR) > $50 \mathrm{cc}$; known allergy to the active substance; patients who had recently undergone ( $<4$ weeks) oral or parenteral antibiotics treatment or who were using prophylactic antibiotic treatment ( $<4$ weeks); positivity to Chlamydia Trachomatis test, Ureaplasma Urealyticum, Neisseria Gonorrhoeae, Herpes Virus (HSV 1/2) and Human Papillomavirus (HPV). Patients were randomized into two arms: one had flower pollen extract, two tablets in a single daily dose for four weeks in line with the previous study of Cai et al. (10). Each dose contained $1 \mathrm{~g}$ of pollen extract and B1, B6, B2, B 9, B12, and PP vitamins. The remnant patients received quercetin $(500 \mathrm{mg}$ ) complex twice daily for four weeks, in line with the study conducted by Shoskes et al. (15).

Validated Italian versions of NIH-CPSI questionnaires (16) and the International Prostate Symptom (IPSS) scores were administered to each patient. The quality of life was evaluated via a translated version of the QoL (17).

The NIH-CPSI was used to determine the effectiveness of clinical therapy (18).

In particular, the expected mean difference for NIH-CPSI was chosen as the primary endpoint.

All the specimen were collected during the urological examination and brought to the laboratory under refrigerated conditions, for cultures, DNA extraction and polymerase chain reaction for Chlamydia Trachomatis, Neisseria Gonorrhoeae, HSV 1/2, and HPV detection.

In addition all the subjects included in the study underwent rectal examination, transrectal ultrasonography, urine and semen cultures, colonies count, and antibiogram if needed (Figure 1).

\section{Statistical analysis}

The main statistical analysis was performed on the patient population "Intent-To-Treat", corresponding to all the ran-

Table 1.

Calculation of the sample size.

\begin{tabular}{|lc|}
\hline Alpha level & 0.05 \\
\hline Power & $90 \%$ \\
\hline Observed average score & 11 \\
\hline Expected average delta & 13 \\
\hline Standard deviation & 2.2 \\
\hline $\mathrm{N}$ & $54(27$ per arm $)$ \\
\hline
\end{tabular}

Table 2.

Patients enrolled in the study.

\begin{tabular}{|lc|}
\hline Number of patients & $\mathbf{5 4}$ \\
\hline Age & $33 \pm 5.25$ \\
\hline Condition duration & $18.85 \pm 4.17$ \\
\hline NIH-CPSI TO & $25.81 \pm 1.61$ \\
\hline IPSS TO & $8.39 \pm 1.74$ \\
\hline QOL TO & $0.55 \pm 0.1$ \\
\hline
\end{tabular}

domized patients described in the CONSORT diagram (Figure 1), a flowchart including the patients evaluated, randomized, analysed and excluded (for any reason).

The baseline patient characteristics are reported as mean, standard deviation (SD), median, inter- quartile range (IQR), frequency, or relative percentage, depending on the type of variable distribution. For the baseline comparison (TO) in the two arms of the study we utilized the T-test for the independent variables and the comparisons of mean values. The chi-square was instead used for the comparison of proportions. The comparison of the mean values of the NIH-CPSI index between baseline (T0) and at 30 days ( $\mathrm{T} 1$ ) was done using paired T-test. All the tests were two-tailed and the statistical significant value was determined to be 0.05 . The sample size was calculated from a starting average waiting time of the variation of the National Institute of Health Chronic Prostatitis Symptom Index (NIH-CPSI) of 13 and a mean observed difference of $11(\mathrm{SD} \pm 2.2)($ Table 1$)$.

\section{Results}

Of the 68 patients presented at our institution with prostatic like symptoms during the study time, 54 were enrolled and randomized. Of the 14 patients excluded, 7 refused the enrolment, 5 were on antibiotic treatment, and 2 reported known allergy to the active substance.

The pre-intervention questionnaires had the following scores: NIH-CPSI $25.81 \pm 1.61$; IPSS $8.39 \pm 1.74$; QoL $0.55 \pm 0.1$ (Table 2).

Table 3 summarizes the clinical characteristics and the enrolment data of the samples taken by arm. There was a statistically significant difference in the duration of the symptoms pre-treatment, and a positive trend in the QoL. There were no other statistically significant differences. At one month follow up the observed results between patients that had taken the flower pollen extract were as follows: NIH-CPSI $12.22 \pm 1.84$, IPSS $7.3 \pm 1.54$, QoL $0.66 \pm 0.1$. Whereas the results for patients that had taken quercetin at the same interval were: NIH-CPSI $14.85 \pm$ 1.85, IPSS $7.67 \pm 1.27$, QoL $0.59 \pm 0.1$ (Table 4).

The improvement of the symptoms after taking flower pollen extract was statistically significant as compared to them taking quercetin $(\mathrm{p}=<0.0001)$. The QoL score was also statistically superior in those taking flower pollen extract $(p=0.003)$. On the other hand, the IPSS score was similar among the two groups $(p=0.39)$. At the follow up all patients had a negative Meares-Stamey test, and showed similar laboratory values, as compared to the initial parameters. No adverse reactions have been reported from the treatment in either arms. The expected value ( $\Delta$ NIH-CPSI), depicted in Chart I and II, obtained by evaluating the expected mean difference for the two arms of the NIH-CPSI pre and post treatment, was of $13.4444 \pm 2.55$ for the flower pollen extract , and of $11.11 \pm 2.69$ for the quercetin, being statistically significant $(\mathrm{p}=0.0019)$ (Figure 1$)$.

\section{Discussion}

The main result of this study is the efficacy of the flower pollen extract in improving the quality of life and 
Table 3.

Clinical characteristics and enrollment data of the samples taken by arm.

\begin{tabular}{|lccc|}
\hline Number of patients & $\begin{array}{c}\text { DEPROX 500 } \\
\text { group }\end{array}$ & $\begin{array}{c}\text { Quercitina } \\
\text { group }\end{array}$ & p value \\
\hline Age & 27 & 27 & - \\
\hline Smokers (\%) & $34 \pm 5.9$ & $33.7 \pm 4.62$ & 0.81 \\
\hline Condition duration & $17.56 \pm 3.88$ & $20.15 \pm 4.11$ & 0.02 \\
\hline NIH-CPSI T0 & $25.67 \pm 1.62$ & $25.96 \pm 1.63$ & 0.5 \\
\hline IPSS T0 & $8.3 \pm 2.13$ & $8.5 \pm 1.3$ & 0.7 \\
\hline QoL T0 & $0.53 \pm 0.1$ & $0.58 \pm 0.1$ & 0.07 \\
\hline Dysuria & $11(40.8 \%)$ & $12(44.5 \%)$ & 0.95 \\
Urgency & $6(22.2 \%)$ & $5(18.5 \%)$ & \\
Dysuria+frequency & $4(14.8 \%)$ & $3(11 \%)$ & \\
Burning sensation & $6(22.2 \%)$ & $7(26 \%)$ & \\
\hline Perineal pain & $12(44 . .5)$ & $7(26 \%)$ & 0.54 \\
Scrotal pain & $5(18.5)$ & $8(30 \%)$ & \\
Soprapubic pain & $5(18.5)$ & $6(22 \%)$ & \\
Lower abdominal pain & $5(18.5)$ & $5(18 \%)$ & \\
No pain & & $1(4 \%)$ & \\
\hline Erectile dysfunction (ED) & $12(44 \%)$ & $10(37 \%)$ & 0.94 \\
Pramture ejaculation PE) & $3(11 \%)$ & $3(11 \%)$ & \\
DE+PE & $4(15 \%)$ & $4(15 \%)$ & \\
No sexual symptoms & $8(30 \%)$ & $10(37 \%)$ & \\
\hline Type Illa & $13(48 \%)$ & $11(41 \%)$ & 0.58 \\
Type Illlb & $14(52 \%)$ & $16(29 \%)$ & \\
\hline
\end{tabular}

Table 4.

1 month follow up.

\begin{tabular}{|lccc|}
\hline $\begin{array}{l}\text { Variables } \\
\text { (after } \mathbf{3 0} \text { days of therapy) }\end{array}$ & $\begin{array}{c}\text { DEPROX 500® } \\
\text { group }\end{array}$ & $\begin{array}{c}\text { Quercitina } \\
\text { group }\end{array}$ & p value \\
\hline NIH-CPSI T1 & $12.22 \pm 1.84$ & $14.85 \pm 1.85$ & $<0.0001$ \\
\hline IPSS T1 & $7.3 \pm 1.54$ & $7.67 \pm 1.27$ & 0.39 \\
\hline QoL T1 & $0.66 \pm 0.1$ & $0.59 \pm 0.1$ & 0.003 \\
\hline
\end{tabular}

reduce pain in patients with CP/CPPS in a statistically significant way compared to the bioflavonoids complex with quercetin. This therapeutic improvement holds true for both the type IIIa and IIIb CP/CPPS patients. Those results are in line with the current literature showing the reduction of pain after 30 days of use of flower pollen extract compared to quercetin (10). This effect is probably secondary to the association between the pollen extract and the vitamins B6 and B12, which enhances the protective effects of the pollen extract on the nerves.

In fact, as demonstrated in animal experiments, the vitamin B complex (including B1, B6, and B12) has analgesic effects in acute and chronic pain secondary to electrical and thermal stimulation, primary and post diabetic neuronal damage $(20,21)$. Several studies have demonstrated that certain vitamin B, B6 and B12 in particular, are capable of protecting the neurons from specific lesions $(22,23)$. Vitamin B1, B6, and B12 are effective in the treatment of painful syndromes such has lumbago, sciatic nerve neuralgia, trigeminal neuralgia, and the chronic pain associated with diabetic polyneuropathy (24).

In contrast to previous studies, our results show that there was no difference in either treatment arms between patient with non-inflammatory CP/CPPS and inflammatory $\mathrm{CP} / \mathrm{CPPS}$. Both the flower pollen extract and the quercetin were well tolerated for the entire duration of the protocol. The limitations of this study are the small number of patients enrolled, a short follow up period, and the selected nature of the patients enrolled. Furthermore, this was not set up as a double blind study. In the absence of a therapeutic efficacy of the "conventional" medications, the therapeutic options with nutritional supplements are a valid alternative. In deciding the therapeutic intervention it is necessary to choose the active principle that determines an improvement of the QoL, the reduction of pain, with high safety levels.

\section{Conclusions}

Considering the above mentioned limitations, the flower pollen extract determined a significant improvement of the symptoms (pain, and quality of life) in the patients with CP/CPPS. A statistically significant difference was also noted in the expected value of the National Institute of Health Chronic Prostatitis Symptom Index ( $\Delta$ NIH-CPSI) in the flower pollen extract group as compared to the Bioflavonoids complex with quercetin, without side effects.

\section{REFERENCES}

1. Krieger JN, et al. NIH consensus definition and classification of prostatitis. JAMA. 1999; 282:236-7

2. Bartoletti $R$, et al. Italian prostatis study group. Prevalence, incidence estimation, risk factors and characterization of chronic prostatitis/ chronic pelvic pain syndrome in urological hospital outpatients in Italy: results of a multicenter case-control observational study. J Urol. 2007; 178:2411-5

\section{Stamey TA. Prostatitis. J R Soc Med. 1981; 74:22-40.}

4. Wagenlehner FM, Weidner W, Naber KG. Therapy for prostatitis, with emphasis on bacterial prostatitis. Expert Pin Pharmacother. 2007; 8:1667-74.

5. Nickel JC. Treatment of chronic prostatitis/chronic pelvic pain syndrome. Int J Antimicrob Agents. 2008; 31 (Suppl 1):S112-6.

6. Herati AS, Moldwin RM. Alternative therapies in the management of chronic prostatitis/chronic pelvic pain syndrome. World J Urol. 2013; 31:761-766.

7. Shoskes DA, Zeitlin SI, Shahed A, Rajfer J. Quercetin in men with category III chronic prostatitis: a preliminary prospective, doubleblind, placebo-controlled trial. Urology. 1999; 54:960-963.

8. Rugendorff EW, Weidner W, Ebeling L, Buck AC. Results of treatment with pollen extract (Cernilton $N$ ) in chronic prostatitis and prostatodynia. Br J Urol. 1993; 71:433-438.

9. Kamijo T, Sato S, Kitamura T. Effect of cernitin pollen-extract on experimental nonbacterial prostatitis in rats. Prostate. 2001; 49:122-131.

10. Cai T, Luciani LG, Caola I, et al. Effects of pollen extract in association with vitamins (DEPROX $\left.500^{\circledR}\right)$ for pain relief in patients affected by chronic prostatitis/chronic pelvic pain syndrome: results from a pilot study. Urologia. 2013; 80(Suppl 22):5-10. 
11. Shoskes DA, Nickel JC. Quercetin for chronic prostatitis/chronic pelvic pain syndrome. Urol Clin North Am. 2011; 38:279-84.

12. Propert KJ, Alexander RB, Nickel JC, et al. Chronic Prostatitis Collaborative Research Network. Design of a multicenter randomized clinical trial for chronic prostatitis/chronic pelvic pain syndrome. Urology. 2002; 59:870-876.

13. Grabe M, Bjerklund-Johansen TE, Botto H, et al. Guidelines on Urological Infections. European Association of Urology; Arnhem, The Netherlands: 2012. p. 66.

14. Wagenlehner FM, Schneider $H$, Ludwig M, et al. A pollen extract (Cernilton) in patients with inflammatory chronic prostatitis-chronic pelvic pain syndrome: a multicentre, randomised, prospective, double-blind, placebo-controlled phase 3 study. Eur Urol. 2009; 56:544-551.

15. Shoskes DA. Quercetin in men with category III chronic prostatitis: a preliminary prospective, double-blind, placebo- controlled trial. Urology. 1999; 54:960-963.

16. Giubilei G, Mondaini N, Crisci A, et al. The Italian version of the National Institutes of Health Chronic Prostatitis Symptom Index. Eur Urol. 2005; 47:805-811.

17. Kaplan RM, Bush JW, Berry CC. Health status: types of validity and the index of well-being. Health Serv Res. 1976; 11:478-507.

18. Nickel JC, Downey J, Hunter D, Clark J. Prevalence of prostatitis-like symptoms in a population based study using the National
Institutes of Health chronic prostatitis symptom index. J Urol. 2001; 165:842-845.

19. Sharma $V$, et al. Sensitization of androgen refractory prostate cancer cells to anti-androgens through re-expression of epigenetically repressed androgen receptor - Synergistic action of quercetin and curcumin. Mol Cell Endocrinol. 2016; 431:12-2.

20. Jolivalt CG, Mizisin LM, Nelson A, et al. B vitamins alleviate indices of neuropathic pain in diabetic rats. Eur J Pharmacol. 2009; 612:41-47.

21. Yu CZ, Liu YP, Liu S, et al. Systematic administration of B vitamins attenuates neuropathic hyperalgesia and reduces spinal neuron injury following temporary spinal cord ischaemia in rats. Eur J Pain. 2014; 18:76-85.

22. Wang ZB, Gan Q, Rupert RL, et al. Thiamine, pyridoxine, cyanocobalamin and their combination inhibit thermal, but not mechanical hyperalgesia in rats with primary sensory neuron injury. Pain. 2005; 114:266-277.

23. Hung KL, Wang CC, Huang CY, Wang SJ. Cyanocobalamin, vitamin B12, depresses glutamate release through inhibition of voltage-dependent $\mathrm{Ca} 2+$ influx in rat cerebrocortical nerve terminals (synaptosomes) Eur J Pharmacol. 2009; 602:230-237.

24. Mäder R, Deutsch H, Siebert GK, et al. Vitamin status of inpatients with chronic cephalgia and dysfunction pain syndrome and effects of a vitamin supplementation. Int J Vitam Nutr Res. 1988; 58:436-441.

\section{Correspondence}

Angela Maurizi, MD (Corresponding Author)

angmau81@hotmail.com

Francesco De Luca, MD

francescodeluca10@gmail.com

Costantino Leonardo, MD

Michele Guidotti, MD

F.P. Antonaccio, MD

Carlo De Dominicis, MD, Professor

Dipartimento di Scienze Ginecologico-ostetriche e Scienze Urologiche,

Università Sapienza Viale dell'Università, 31/33, 00161, Roma, Italy

Antonino Zanghì, MD

Dipartimento di Scienze Mediche, Chirurgiche e Tecnologie Avanzate

G.F. Ingrassia, Università di Catania, Catania, Italy

Emy Manzi, MD

emymanzi@gmail.com

Via San Pasquale, 03043 Cassino (FR), Italy

Valerio Olivieri, MD

valerio.olivieri@uniromal.it

Piazza Credenza 2, 10015 Ivrea (TO), Italy 